\title{
Intraoperative high-resolution ultrasound and contrast-enhanced ultrasound of peripheral nerve tumors and tumorlike lesions
}

\author{
Maria Teresa Pedro, MD, ${ }^{1}$ Gregor Antoniadis, MD, PhD, ${ }^{1}$ Angelika Scheuerle, MD, ${ }^{2}$ \\ Mirko Pham, MD, PhD, ${ }^{3}$ Christian Rainer Wirtz, MD, PhD, ${ }^{1}$ and Ralph W. Koenig, MD, PhD1 \\ Departments of ${ }^{1}$ Neurosurgery and ${ }^{2}$ Neuropathology, University of Ulm, Guenzburg; and ${ }^{3}$ Department of Neuroradiology, \\ University of Heidelberg, Germany
}

\begin{abstract}
The diagnostic workup and surgical therapy for peripheral nerve tumors and tumorlike lesions are challenging. Magnetic resonance imaging is the standard diagnostic tool in the preoperative workup. However, even with advanced pulse sequences such as diffusion tensor imaging for MR neurography, the ability to differentiate tumor entities based on histological features remains limited. In particular, rare tumor entities different from schwannomas and neurofibromas are difficult to anticipate before surgical exploration and histological confirmation. High-resolution ultrasound (HRU) has become another important tool in the preoperative evaluation of peripheral nerves. Ongoing software and technical developments with transducers of up to $17-18 \mathrm{MHz}$ enable high spatial resolution with tissue-differentiating properties. Unfortunately, high-frequency ultrasound provides low tissue penetration. The authors developed a setting in which intraoperative HRU was used and in which the direct sterile contact between the ultrasound transducer and the surgically exposed nerve pathology was enabled to increase structural resolution and contrast. In a case-guided fashion, the authors report the sonographic characteristics of rare tumor entities shown by intraoperative HRU and contrast-enhanced ultrasound.
\end{abstract}

http://thejns.org/doi/abs/10.3171/2015.6.FOCUS15218

KEY WORDS high-resolution ultrasound; intraoperative setting; nerve tumor; tumorlike lesions; contrast-enhanced ultrasound; sonographic characteristics

$\mathrm{P}$ ERIPHERAL nerve tumors are not uncommon. Schwannomas and neurofibromas, for instance, each account for $5 \%$ of all soft-tissue tumors. ${ }^{13}$ Their diagnostic and surgical management may be demanding at many times, mainly because it is difficult to anticipate the true histological differentiation of the lesion before surgery and because surgical preparation and resection carry the risk for loss of nerve function. Peripheral nerve tumors are usually first recognized on clinical examination and subsequent MRI. Most often, these tumorous lesions will correspond to schwannomas or neurofibromas of peripheral nerves. However, the accurate presurgical determination of rare and, in particular, malignant tumor entities such as malignant peripheral nerve sheath tumors (MPNSTs) remains insufficient even when advanced methods such as MRI (so-called MR neurography enhanced by, e.g., diffusion tensor imaging) or PET are used. For instance, no definitive differentiation between MPNSTs and plexiform neurofibromas is possible yet with any means. ${ }^{9}$

Other extremely rare nerve tumors or tumorlike lesions, such as perineurioma, amyloidoma, lymphoma, $4,7,8,18$ and multifocal acquired demyelinating sensory and motor neuropathy (MADSAM), are even harder to distinguish preoperatively. ${ }^{20}$ However, because the management of these conditions differs widely, ${ }^{9,19,21}$ a reliable diagnosis is crucial.

ABBREVIATIONS CEUS = contrast-enhanced ultrasound; HRU = high-resolution ultrasound; i = intraoperative; LSS = Lewis Sumner syndrome; MADSAM = multifocal acquired demyelinating sensory and motor neuropathy; MPNST = malignant peripheral nerve sheath tumor; NF-1 = neurofibromatosis Type 1; SFT = solitary fibrous tumor. SUBMITTED May 1, 2015. ACCEPTED June 12, 2015. 
Since the late $1980 \mathrm{~s},{ }^{10}$ the application of ultrasound in soft tissues has played an important role. Focusing on peripheral nerves, high-resolution ultrasound (HRU) is mainly applied in the preoperative workup of nerve entrapment and trauma. For traumatic lesions, nerve transection or hemorrhage is safely depicted by ultrasound, which enables nerve surgeons to perform nerve reconstruction earlier than ever before. ${ }^{11}$ In 2011, our study group examined traumatic nerve lesions in an intraoperative setting by using the full technical capacity of HRU with its very high spatial resolution and tissue-differentiating properties. ${ }^{12}$ By this means, the nerve is externally neurolyzed and embedded between 2 ultrasound gel cushions, and the transducer is set directly on the nerve segment to be evaluated. This technique enables the surgeon to see directly into the fascicular structure and epineurium.

Furthermore, the technique of contrast-enhanced ultrasound (CEUS) was developed as a tool for differentiating benign and malignant pathologies in the liver or kidney.,22 In the present study, we investigated intraoperative HRU and CEUS (iHRU and iCEUS, respectively) with regard to the question of whether these techniques have the potential to help the surgeon to distinguish different peripheral nerve tumors and tumorlike lesions.

\section{Methods}

From May 2010 to October 2014, a total of 161 patients underwent surgery for peripheral nerve tumors in our department. As part of the preoperative diagnostic workup, each patient underwent MRI, and if malignancy was suspected, additional FDG PET and PET CT scans were performed. In 13 cases (8\%), this preoperative workup revealed "atypical" signs of nerve tumors, meaning that the tumors were something other than schwannoma or neurofibroma. To gain further morphological knowledge in those cases, iHRU was performed.

The intraoperative ultrasound examination (iU22, Philips Medical Systems) was carried out using a sterilely draped small-footprint 15- to 7-MHz (Fig. 1 upper and lower) or alternatively a $17-$ to $5-\mathrm{MHz}$ linear array ultrasound transducer. Depending on their properties in iHRU, groups of ultrasound patterns were defined and correlated to the final histopathological diagnosis. In 3 cases, additional iCEUS was applied to study tumor perfusion using a 12-MHz probe. As a contrast medium, 1 ampule of SonoVue (Bracco Imaging) with $5 \mathrm{ml}$ saline solution was administered intravenously.

\section{Results}

The study group consisted of 7 male and 6 female patients ranging in age from 23 months to 56 years (mean age 36 years). Histopathological results were available an average of 3-7 days after surgery. A total of 7 different types of tumors or tumorlike lesions (perineurioma $[\mathrm{n}=$ 4], amyloidoma [ $\mathrm{n}=2$ ], Lewis Sumner syndrome [LSS] $[\mathrm{n}=1]$, B-cell lymphoma [ $\mathrm{n}=1]$, MPNST [ $=3$ ], extrapleural solitary fibrous tumor [SFT] $[\mathrm{n}=1]$, and ancient schwannoma $[\mathrm{n}=1]$ ) were examined (Table 1). Reference pathology was performed in 4 cases (for the patients with LSS or MPNST). The examination took up to 5 minutes to
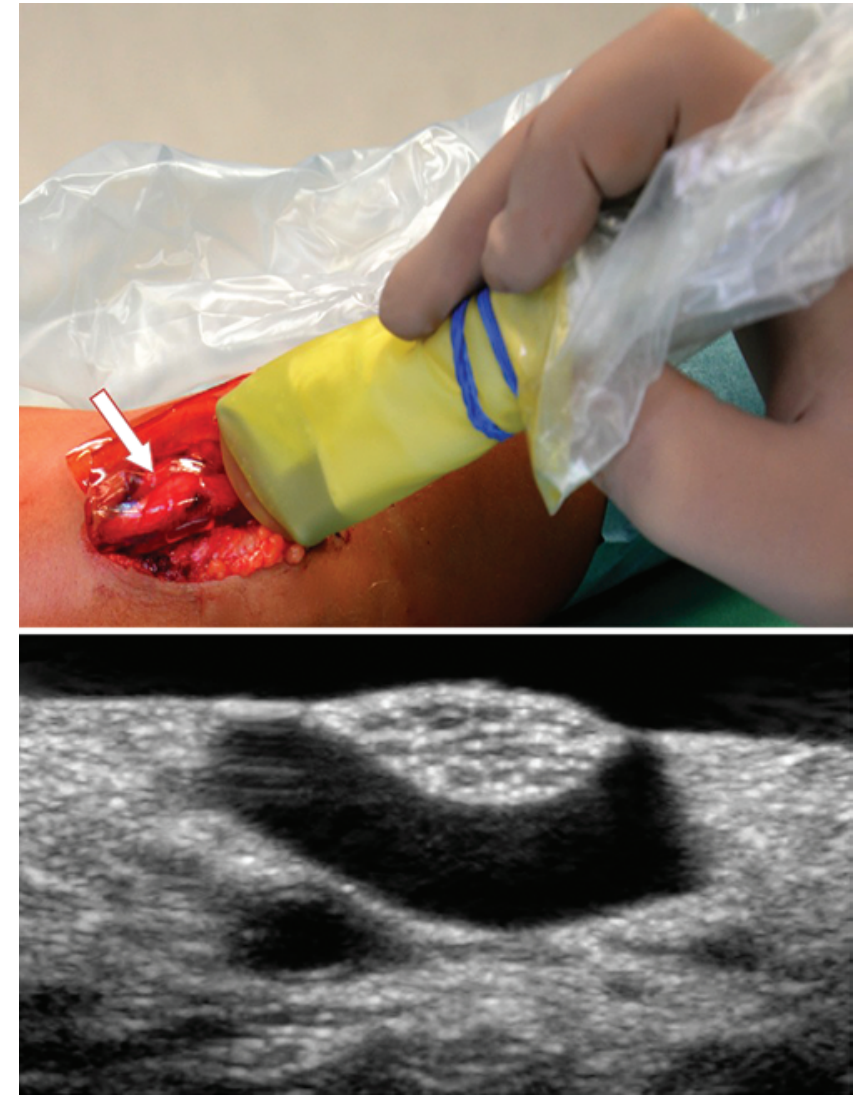

FIG. 1. Upper: iHRU being performed with a sterile 15- to 7-MHz hockey-stick probe. The ulnar nerve of the left elbow is embedded in 2 sterile gel cushions (white arrow). Lower: The corresponding ultrasound image is shown, depicting fine hypoechoic fascicles of the ulnar nerve without pathological findings at the region.

determine the respective perfusion pattern of each tumor (Table 2).

Excluding the patients with an MPNST, each patient had paresthesia and slowly progressing palsy in the distribution of the respective nerve.

Depending on their ultrasonographic peculiarities in iHRU, 3 different ultrasound patterns were found in the study group, and each was assigned to 1 of the following 3 different pathological descriptions: 1) hypoechoic, enlarged fascicles (perineurioma, amyloidoma, LSS) (Group A); 2) dense, giant fascicles (B-cell lymphoma) (Group $\mathrm{B})$; or 3) no fascicle structure distinguishable, irregular mass, cystic, hypoechoic or isoechoic to hyperechoic parts (MPNST, ancient schwannoma, SFT) (Group C).

\section{Group A: Perineurioma, Amyloidoma, and LSS}

Although of different origins and pathologies, perineurioma, amyloidoma, and LSS resembled each other in iHRU; the nerves' cross-sections were markedly enlarged and contained large, thickened hypoechogenic fascicles. The epineuria appeared normal. On closer examination, subtle details distinguished the different entities from each other (Figs. 2-4): in cases of perineurioma and amyloidoma, hypoechogenic enlarged fascicles were detected throughout the entire cross-section of each nerve, and in 
TABLE 1. Descriptive statistics, anatomical tumor location, diagnosis, and preoperative diagnostic imaging type in patients in the study group

\begin{tabular}{|c|c|c|c|c|c|}
\hline Patient No. & Sex, Age & Nerve & Diagnosis & MRI & FDG PET \& PET CT \\
\hline 1 (Fig. 4$)^{*}$ & $\mathrm{~F}, 39 \mathrm{yrs}$ & Nervus ischiadicus & LSS & $x$ & \\
\hline 2 (Fig. 5$)^{*}$ & $F, 56$ yrs & Nervus ischiadicus & B-cell lymphoma & $x$ & \\
\hline 3 & M, 31 yrs & Nervus ischiadicus & MPNST (WHO Grade IV) & $x$ & $x$ \\
\hline 4 (Fig. 2) ${ }^{*}$ & $\mathrm{~F}, 10 \mathrm{yrs}$ & Nervus ischiadicus & Perineurioma & $x$ & \\
\hline 5 & $\mathrm{M}, 23 \mathrm{mos}$ & Nervus ischiadicus & Perineurioma & $x$ & \\
\hline 6 & $\mathrm{~F}, 55 \mathrm{yrs}$ & Nervus peroneus communis & Amyloidoma & $x$ & \\
\hline 7 (Fig. 3) ${ }^{*}$ & F, 43 yrs & Nervus ulnaris & Amyloidoma & $x$ & \\
\hline 8 & M, 39 yrs & Nervus medianus & Perineurioma & $x$ & \\
\hline 9 & M, 29 yrs & Plexus brachialis & Perineurioma & $x$ & \\
\hline $10(\text { Fig. 6) })^{*}$ & M, 29 yrs & Nervus peroneus communis & MPNST (WHO Grade IV) & $x$ & $x$ \\
\hline 11 & M, 45 yrs & Nervus tibialis & Extrapleural SFT & $x$ & \\
\hline 12 (Fig. 7)* & M, 56 yrs & Posterior division of plexus brachialis & Ancient schwannoma & $x$ & \\
\hline 13 (Fig. 8) $)^{*}$ & $\mathrm{~F}, 39 \mathrm{yrs}$ & Nervus ischiadicus & MPNST (WHO Grade IV) & $x$ & $x$ \\
\hline
\end{tabular}

FDG PET = fluorine-18-labeled fluorodeoxyglucose positron emission tomography; $x=$ performed.

* Case is illustrated in the indicated figure.

cases of LSS, normal fascicles were detected beneath the hypoechogenic and enlarged fascicular structures.

\section{Group B: B-Cell Lymphoma}

The ultrasound pattern in Group B was exceptional: the nerve exhibited a marked cross-sectional enlargement, and the epineurium appeared to be normal. The fascicular structures were markedly enlarged, dense, and hyperechogenic, with hypoechogenic septae subdividing the fascicle groups.

This unusual sciatic nerve tumor at the midthigh level became symptomatic, with slowly progressive paresis including foot elevation and flexion and paresthesia of the sole and dorsal thigh. During surgery after exposure of the nerve and ultrasound, we decided to open the epineurium and perform a biopsy of a conspicuous fascicle. The fascicles appeared to be transparent (Fig. 5A-D). Histopathological diagnosis revealed B-cell lymphoma. After oncological staging, it turned out to be the solitary manifestation of B-cell lymphoma in that female patient. Radiotherapy was performed, but she refused additional chemotherapy; 2.5 years after diagnostic identification, the patient was still alive.

\section{Group C: MPNST, Ancient Schwannoma, and SFT}

Although in their preoperative imaging, all lesions in this group showed similarities, such as positive contrast enhancement in MRI (T1-weighted sequence) and irregular tumor masses in HRU, this group of lesions turned out to be inhomogeneous in their particular histopathological results. Intraoperative ultrasound revealed loss of fascicular structure combined with cystic formations for each of them. The tumor masses consisted of adjacent areas of isoechoic, hypoechoic, and hyperechoic tissue (see an example of an MPNST in Fig. 6).

In the synopsis of the findings, suspicion of potential malignancy was indicated for each of the patients in this group $(n=5)$. With the objective of further improving tis-

TABLE 2. Overview of results, grouped by histology, iHRU, power Doppler, and perfusion characteristics

\begin{tabular}{|c|c|c|c|}
\hline Nerve Tumor Type & iHRU Results & $\begin{array}{l}\text { Power Doppler } \\
\quad \text { Results }\end{array}$ & CEUS Results \\
\hline Perineurioma $(n=4)$ & Hypoechoic, thickened definable fascicles & - & Not performed \\
\hline Amyloidoma $(n=2)$ & Hypoechoic, thickened fascicles; sizes varied & - & Not performed \\
\hline $\operatorname{LSS}(n=1)$ & $\begin{array}{l}\text { Hypoechoic, thickened fascicles; not involving complete } \\
\text { nerve }\end{array}$ & - & Not performed \\
\hline B-cell lymphoma $(n=1)$ & Isoechoic dense giant fascicles & - & Not performed \\
\hline MPNST $(n=3)$ & $\begin{array}{l}\text { Irregular; isoechoic or hyperechoic to hypoechoic; cystic } \\
\text { w/ solid parts }\end{array}$ & $-1+$ & Perfusion pattern* \\
\hline Extrapleural SFT $(n=1)$ & $\begin{array}{l}\text { No fascicles distinguishable; irregular isoechoic to } \\
\text { hypoechoic }\end{array}$ & - & No perfusion pattern \\
\hline Ancient schwannoma $(n=1)$ & Cystic; isoechoic to hypoechoic; irregular & - & Perfusion pattern \\
\hline
\end{tabular}



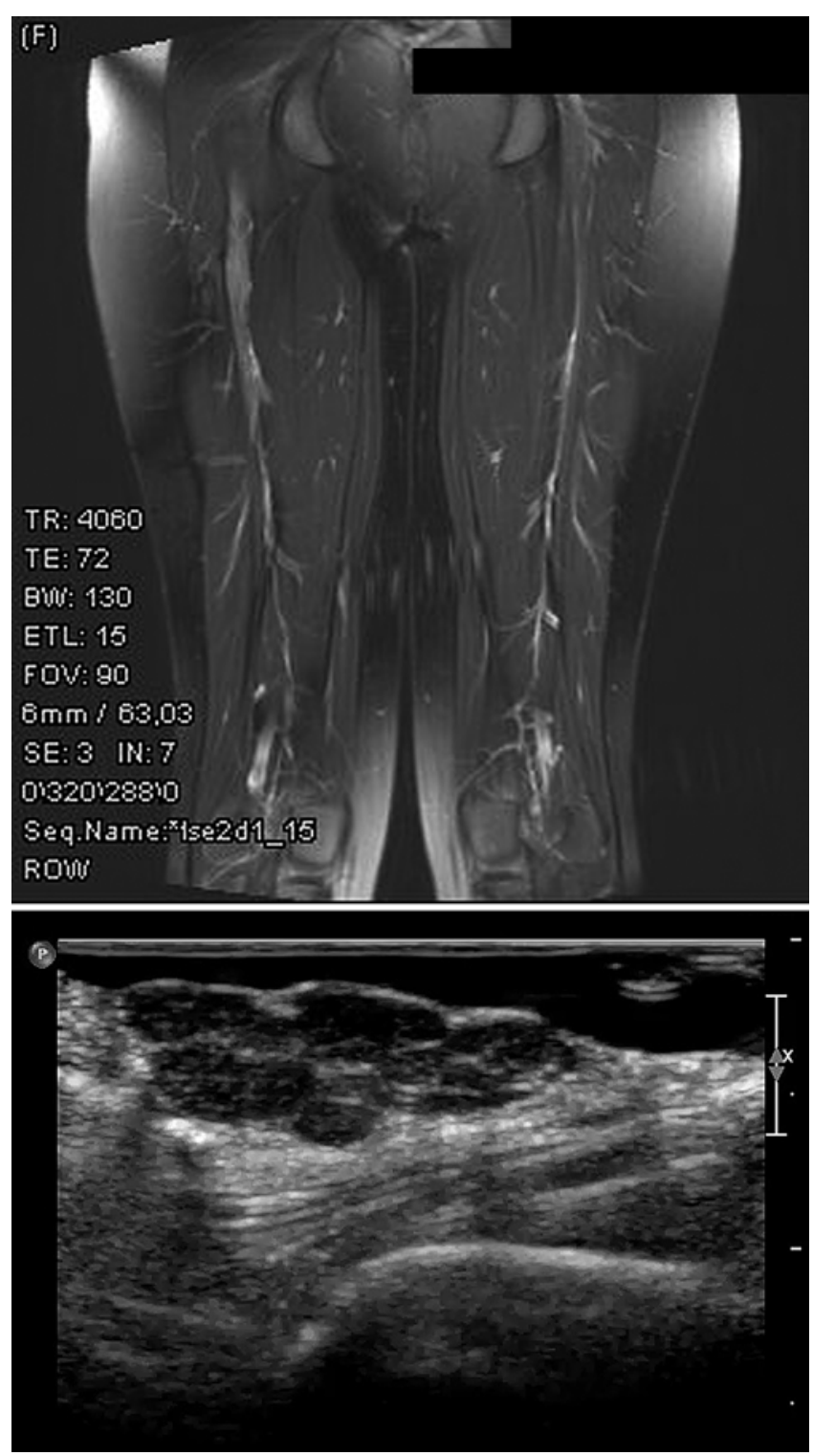

FIG. 2. Patient 4. A turbo-spin echo coronal MR image (upper) showing an enlargement of the right proximal sciatic nerve and iHRU image (lower) of the perineurioma (sciatic nerve) showing large hypoechoic fascicles; the complete nerve is involved.

sue differentiation, iCEUS was applied in 3 of these patients. Thus far, the only available contrast medium, SonoVue, is officially approved only for mammary and liver ultrasound. In peripheral nerve tumors, its use until now has been considered off label. As part of individualized treatment, we used CEUS after the written consent of each patient was obtained.

The first patient (Table 1, Patient 11), who had a slowly growing mass in his lower leg, had minimal neurological deficit. The tumor arose from the tibial nerve. The second patient, who had a brachial plexus tumor (Table 1, Patient 12), suffered from pain radiating into his left upper arm under strain (Fig. 7A-F).

The last case (Table 1, Patient 13) was of a young woman suffering from neurofibromatosis Type 1 (NF-1) with a tumor mass involving her sciatic nerve in the thigh. That lesion has been surgically treated 2 years earlier. Histopathology revealed MPNST. She refused any adjuvant therapy after the first surgery. In the summer of 2014, she developed a fast-growing recurrence and underwent further resection (Fig. 8 upper and lower).

Comparing this last NF-1 case (Patient 13) with that of a brachial plexus tumor (Patient 12), the iHRU images seemed very similar. Moreover, both cases showed positive perfusion patterns on iCEUS images. However, the time to peak perfusion differed markedly in both lesions: 19 seconds in the MPNST case and more than 1 minute in the brachial plexus tumor. Histopathology revealed ancient schwannoma (WHO Grade I) in the patient with the brachial plexus tumor and recurrent MPNST in the NF-1 case.

The other case (Patient 11) resulted in identification of an extrapleural SFT of the tibial nerve, which was classified as French Fédération Nationale des Centres de Lutte Contre le Cancer Grade II. The tumor did not show any perfusion pattern in the iCEUS images.

\section{Discussion}

To our knowledge, this is the first study to use iHRU for tumors and tumorlike nerve lesions. As Rodriguez et al. ${ }^{17}$ already pointed out in their pathological study, the differential diagnosis of these pathologies is difficult and controversial. Because we gained positive experience with the tissue-differentiating properties of iHRU in traumatic nerve lesions, ${ }^{12}$ we decided to apply this technique to tumors and tumorlike lesions of peripheral nerves.

In our limited experience with 13 patients, 3 different patterns have been distinguished in iHRU images so far.

\section{Group A (Hypoechoic, Enlarged Fascicles)}

Hypoechoic swollen fascicles are frequently found in peripheral nerve lesions, mostly in cases of nerve entrapment. ${ }^{11}$ In Group A, we found this pattern in perineuriomas, in amyloidomas, and in 1 case of MADSAM. In our preliminary experience so far, no definitive differentiation between those 3 entities was possible with iHRU. However, a subtle difference between perineuriomas and amyloidomas and MADSAM was noticed: in perineuriomas and amyloidomas, hypoechogenic and enlarged fascicles took up the complete cross-sectional area of the nerve, whereas with MADSAM, normal fascicles were detected beneath those hypoechogenic and enlarged fascicular structures. This interesting finding points toward the multifocal nature of lesion dispersion in this inflammatory neuropathy. ${ }^{20}$

\section{Group B (Dense, Giant Fascicles)}

The iHRU pattern of dense giant fascicles was detected in 1 patient and did not resemble any other ultrasound pattern seen before by us. This ultrasound finding was seen on images of a patient with a very rare entity scarcely described in the literature so far: ${ }^{8}$ primary B-cell lymphoma involving the sciatic nerve. Similar to a description by Descamps et al., ${ }^{8}$ MRI of this patient revealed a contrast-enhancing edematous lesion of the sciatic nerve 


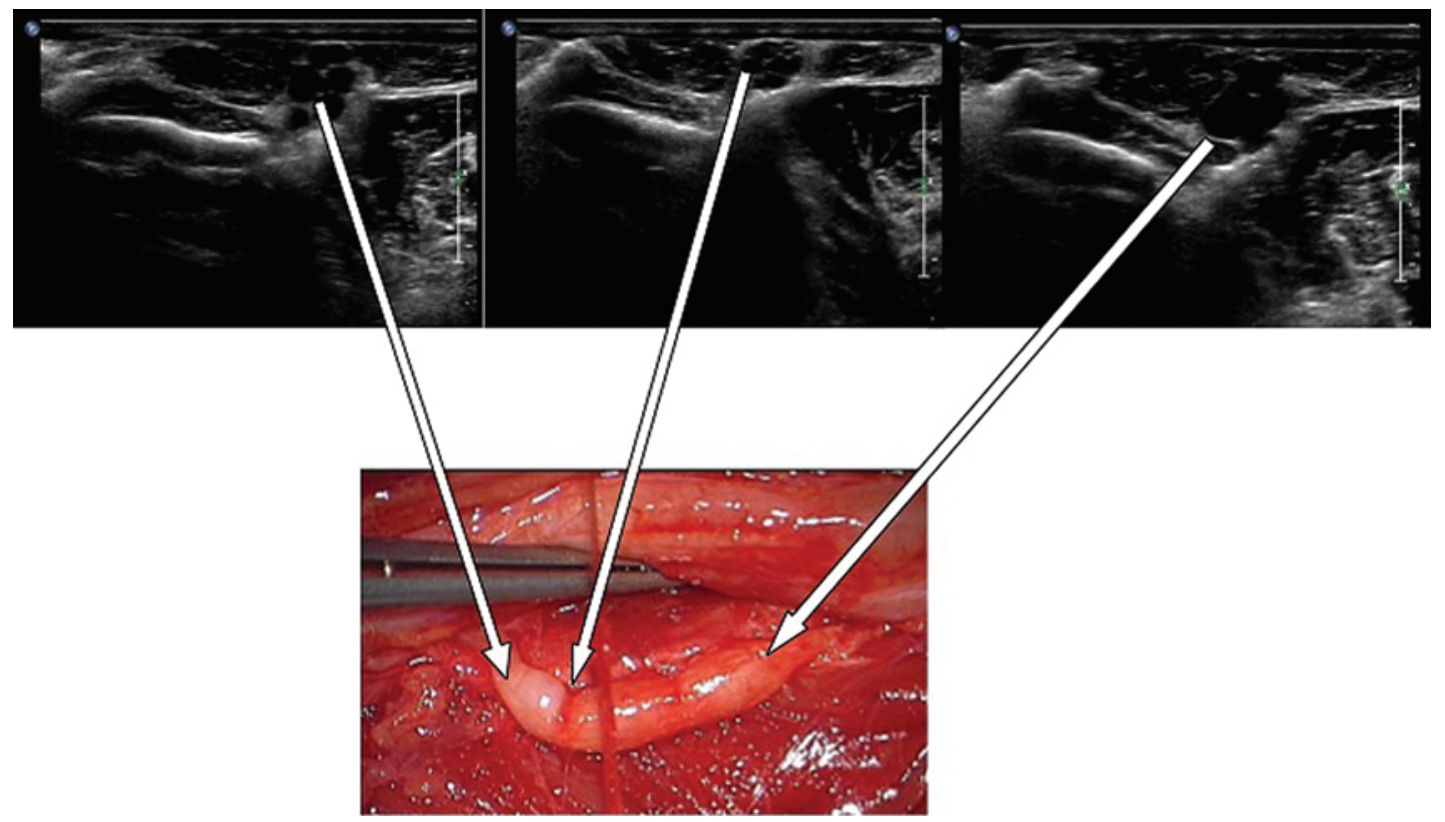

FIG. 3. Patient 7. Upper: iHRU images of the amyloidoma (ulnar nerve) depicting irregular thickened hypoechoic fascicles. Lower: Intraoperative image of 1 fascicle with some parts of it being constricted.

that resembled a perineurioma or amyloidoma. Intraoperative findings and iHRU revealed a unique pathology, well distinguishable from that of all other tumor entities. In a recent study, Capek et al. ${ }^{6}$ described another case of a diffuse large B-cell lymphoma of the sciatic nerve examined by ultrasound. Similar to our findings, nerve fascicles were preserved but enlarged and infiltrated by the hematological disease.

\section{Group C (No Fascicle Structure Distinguishable, Irregular Mass, Cystic)}

This group turned out to be very inhomogeneous. Despite its spatial high resolution with good tissue-differentiating properties, HRU alone was not capable of differentiating pathologies, particularly benign and malignant peripheral nerve tumors.

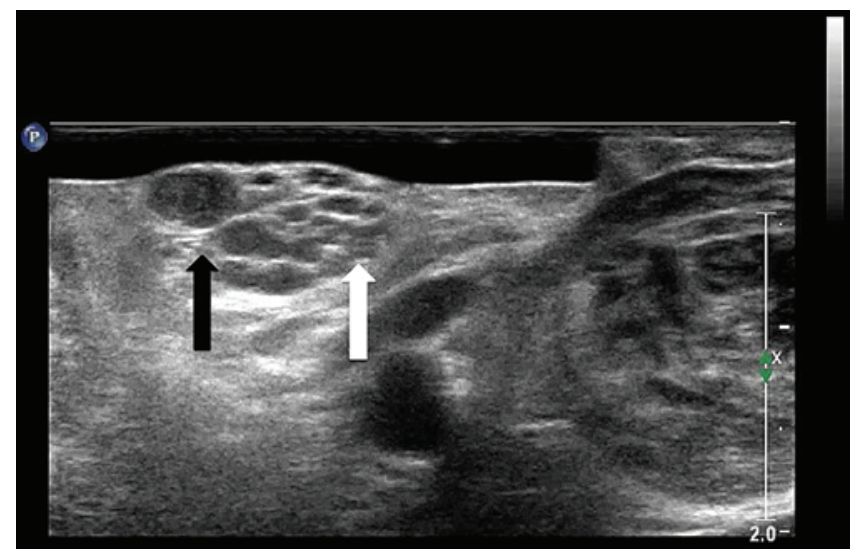

FIG. 4. Patient 1. iHRU of confirmed LSS (sciatic nerve). Note that fascicle enlargement is heterogeneous, with some fascicles more enlarged than others (black arrow vs white arrow).
To acquire more knowledge about potential malignant nerve tumors, we decided to apply SonoVue (Bracco Imaging) as a contrast agent for ultrasonography performed in this study. In 2002, Bodner et al. ${ }^{3}$ proved that additional ultrasound tools such as power Doppler and spectral wave analysis improve the differentiation of malignant and benign musculoskeletal tumors by depicting their vascular architecture. In 2012, Loizides et al. ${ }^{14}$ used CEUS to examine 54 patients with musculoskeletal masses. An irreg-
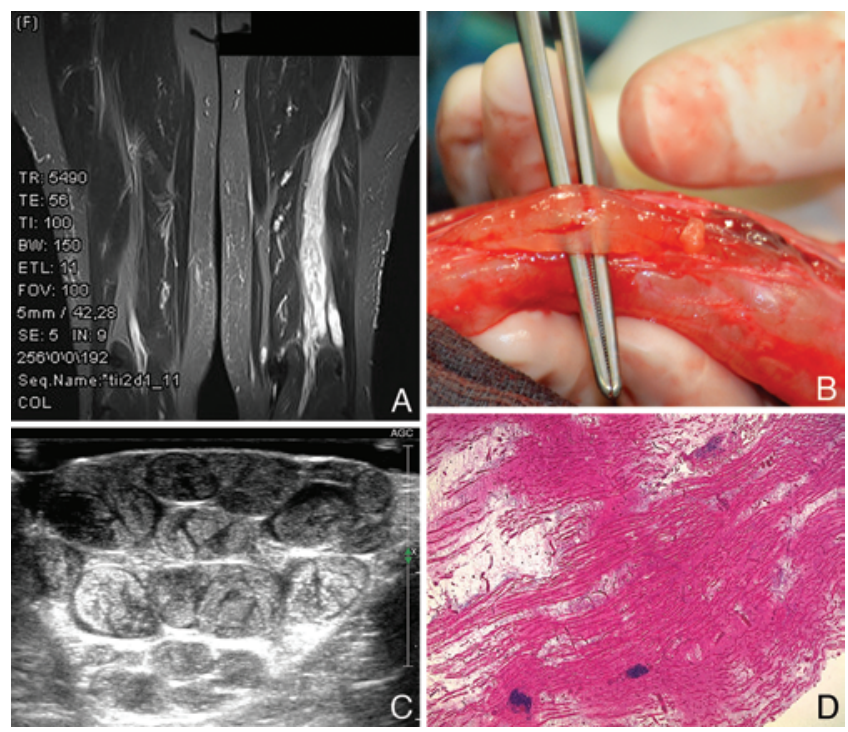

FIG. 5. Patient 2. A: A coronal turbo inversion recovery MR image of both thighs revealing a hyperintense lesion of the left sciatic nerve. B: Intraoperative photograph demonstrating transparent fascicle of the sciatic nerve (forceps). C: In the iHRU image, giant "dense" fascicles can be seen. D: Photomicrograph showing leukemic infiltrate, $\mathrm{H}$ \& $\mathrm{E}$. Tests were positive for tumor markers CD5, CD20, CD23, IgD, and lgM. 


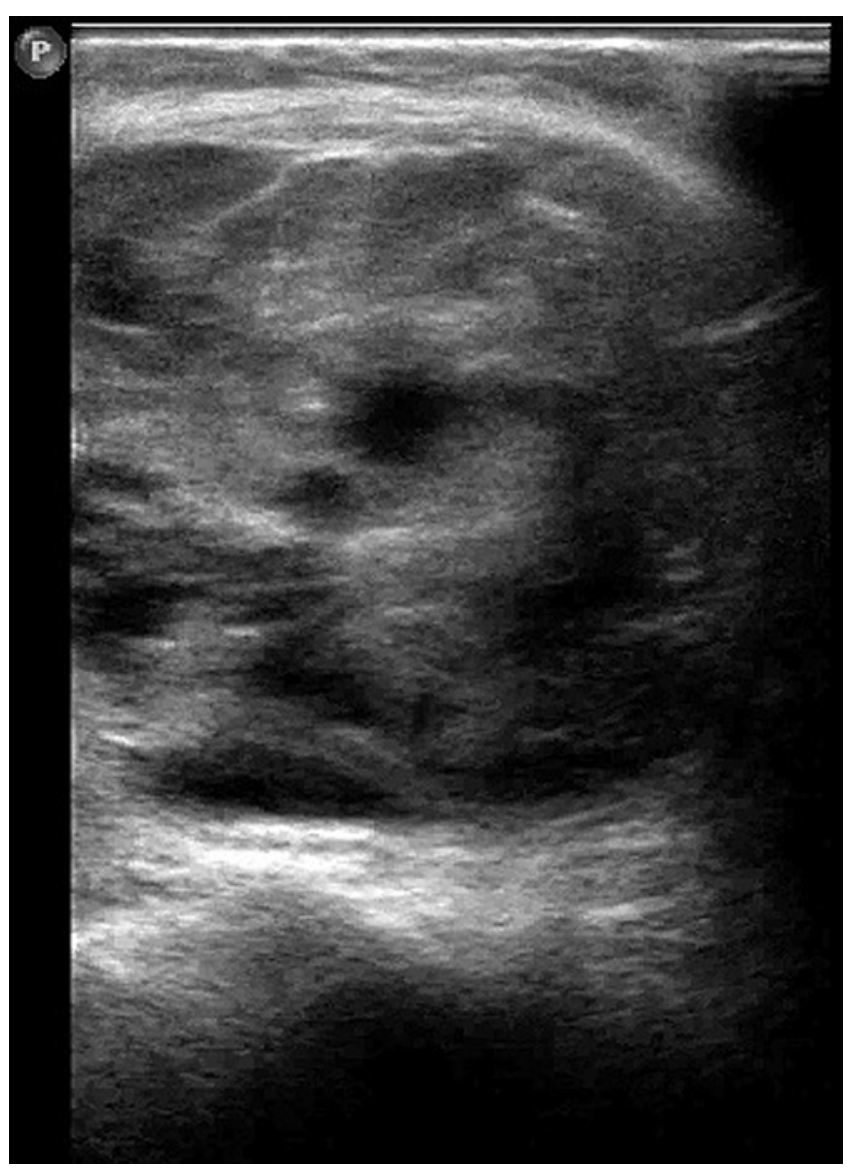

FIG. 6. Patient 10. HRU image of the left peroneal nerve of a patient with NF-1 showing high irregular signs of cystic hypoechoic to solid hyperechoic areas. No determination of fascicular structure is possible. ular positive perfusion pattern, which was determined as Type $\mathrm{P} 2$ or $\mathrm{P} 3$, indicated a high probability of malignancy. Concerning nerve tumors, there was just 1 case of schwannoma with a positive Type P4 perfusion pattern, which was a sign of benignity. Because of the low differentiating properties of ultrasound, especially in Group C, we decided to apply CEUS to explore its capability for enhancing the differential diagnostic distinction. iCEUS was applied in 3 cases: 1 of ancient schwannoma, 1 of SFT, and 1 of MPNST. Although the SFT did not show any perfusion at all, the perfusion pattern of benign cystic schwannoma very much resembled that of the MPNST. However, the time to peak (the time from injection of the contrast agent to its detection) differed significantly between the degenerative modified ancient schwannoma and the MPNST. The MPNST had a very short time to peak.

These results agree with those of many publications regarding ultrasound of other organs (e.g., liver), indicating that, beneath the spatial pattern of perfusion, the temporal course serves as a valuable discriminator. In 2009, a group from Verona, Italy, showed positive enhancement in the arterial phase by examining nonfunctioning pancreatic neuroendocrine tumors. CEUS revealed a rapid positive perfusion pattern in $63.1 \%$ of their patients. Moreover, these results correlated with a positive $\mathrm{Ki}-67$ index and the existence of hepatic metastasis. ${ }^{15}$ After the first results in breast tumors, the application of CEUS was not pathbreaking for the identification of malignancy. ${ }^{2}$ Therefore, in 2011, CEUS was not recommended in the European Federation of Societies for Ultrasound in Medicine and Biology guidelines ${ }^{16}$ for the examination of mamma carcinoma. Meanwhile, in the latest studies from 2014, a correlation between CEUS characteristics and prognostic factors for breast cancer was detected. ${ }^{5}$
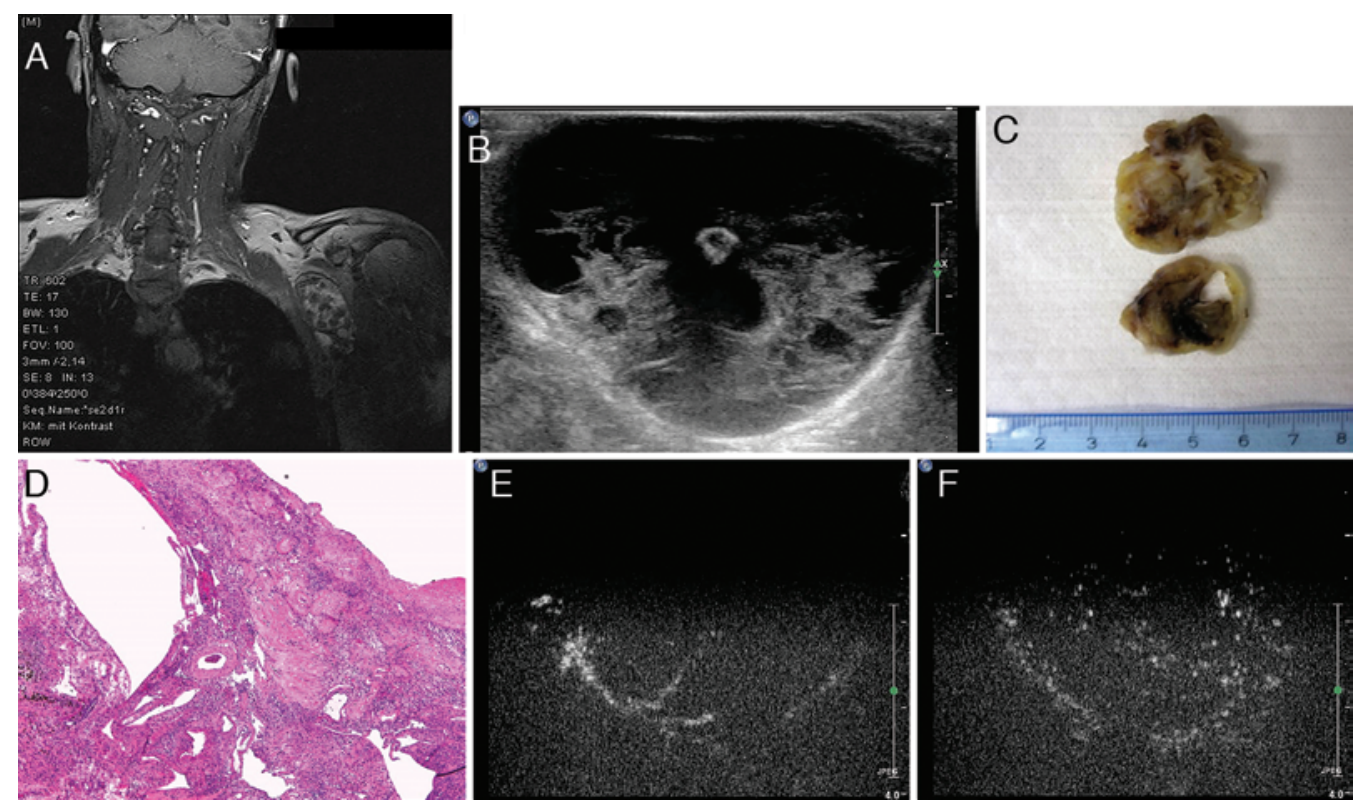

FIG. 7. Patient 12. Coronal T1-weighted Gd-enhanced MR image (A) of the brachial plexus showing an irregular cystic tumor mass of the posterior division and iHRU image (B) (17-5 MHz) showing the corresponding cystic hypoechoic to hyperechoic formation; the nerve fascicles cannot be distinguished. Correlating tumor masses (C) and their pathological image, $\mathrm{H} \& \mathrm{E}$ (D). iCEUS image $(12 \mathrm{MHz}$ ) depicting bubbles (positive perfusion pattern) after 1 minute (E) and accumulating over the next 4 minutes (F). 

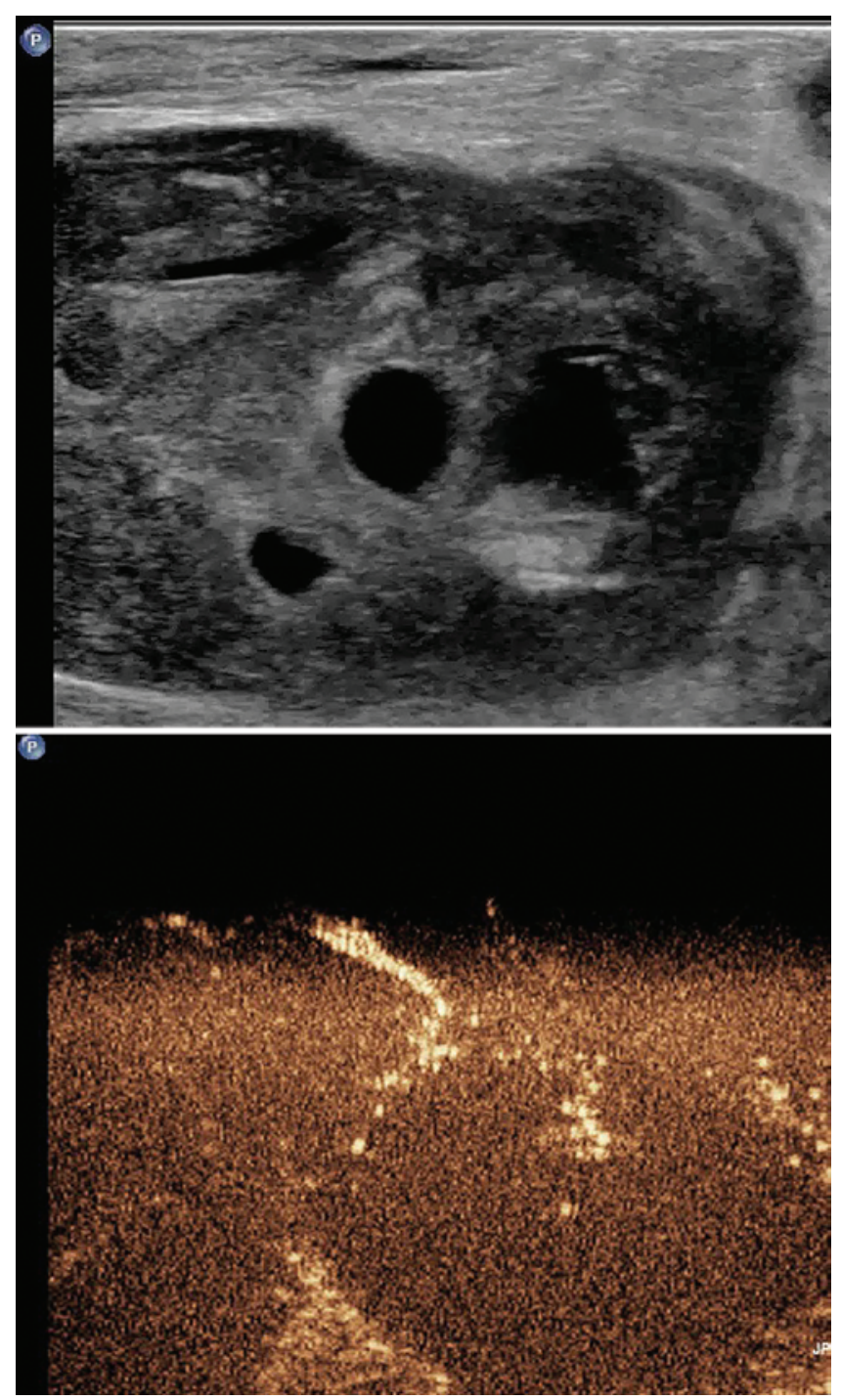

FIG. 8. Patient 13. Upper: iHRU image (12 MHz) showing a cystic irregular hypoechoic to hyperechoic mass of the sciatic nerve. Lower: iCEUS image (12 MHz) demonstrating a positive perfusion pattern after only a 19-second delay following intravenous administration of 1 ampule of SonoVue with $5 \mathrm{ml}$ saline solution and diminishing during the next 60 seconds.

\section{Conclusions}

Multimodal ultrasound seems to be a promising diagnostic tool in the diagnostic differentiation of peripheral nerve tumors and tumorlike lesions. Additional systematic studies are necessary to characterize and differentiate these different pathologies.

\section{References}

1. Arti M, Tatatabaeifar L, Jang HJ, Finelli A, Moshonov H, Jewett M: Accuracy of contrast enhanced ultrasound for differentiating benign from malignant solid small renal masses. Radiology 28:140907, 2015

2. Balleyguier C, Opolon P, Mathieu MC, Athanasiou A, Garbay JR, Delaloge $S$, et al: New potential and applications of contrast-enhanced ultrasound of the breast: Own investiga- tions and review of the literature. Eur J Radiol 69:14-23, 2009

3. Bodner G, Schocke MF, Rachbauer F, Seppi K, Peer S, Fierlinger A, et al: Differentiation of malignant and benign musculoskeletal tumors: combined color and power Doppler US and spectral wave analysis. Radiology 223:410-416, 2002

4. Boyanton BL Jr, Jones JK, Shenaq SM, Hicks MJ, Bhattacharjee MB: Intraneural perineurioma: a systematic review with illustrative cases. Arch Pathol Lab Med 131:1382-1392, 2007

5. Cao XL, Bao W, Zhu SG, Wang LH, Sun MH, Wang L, et al: Contrast-enhanced ultrasound characteristics of breast cancer: correlation with prognostic factors. Ultrasound Med Biol 40:11-17, 2014

6. Capek S, Hébert-Blouin MN, Puffer RC, Martinoli C, Frick MA, Amrami KK, et al: Tumefactive appearance of peripheral nerve involvement in hematologic malignancies: a new imaging association. Skeletal Radiol 44:1001-1009, 2015

7. Consales A, Roncaroli F, Salvi F, Poppi M: Amyloidoma of the brachial plexus. Surg Neurol 59:418-423, 2003

8. Descamps MJ, Barrett L, Groves M, Yung L, Birch R, Murray NM, et al: Primary sciatic nerve lymphoma: a case report and review of the literature. J Neurol Neurosurg Psychiatry 77:1087-1089, 2006

9. Ferner RE, Golding JF, Smith M, Calonje E, Jan W, Sanjayanathan V, et al: $\left[{ }^{18} \mathrm{~F}\right] 2$-fluoro-2-deoxy-D-glucose positron emission tomography (FDG PET) as a diagnostic tool for neurofibromatosis 1 (NF1) associated malignant peripheral nerve sheath tumours (MPNSTs): a long-term clinical study. Ann Oncol 19:390-394, 2008

10. Fornage BD: Peripheral nerves of the extremities: imaging with US. Radiology 167:179-182, 1988

11. Koenig RW, Pedro MT, Heinen CPG, Schmidt T, Richter HP, Antoniadis G, et al: High-resolution ultrasonography in evaluating peripheral nerve entrapment and trauma. Neurosurg Focus 26(2):E13, 2009

12. Koenig RW, Schmidt TE, Heinen CPG, Wirtz CR, Kretschmer T, Antoniadis G, et al: Intraoperative highresolution ultrasound: a new technique in the management of peripheral nerve disorders. J Neurosurg 114:514-521, 2011

13. Kransdorf MJ: Benign soft-tissue tumors in a large referral population: distribution of specific diagnoses by age, sex, and location. AJR Am J Roentgenol 164:395-402, 1995

14. Loizides A, Peer S, Plaikner M, Djurdjevic T, Gruber H: Perfusion pattern of musculoskeletal masses using contrastenhanced ultrasound: a helpful tool for characterisation? Eur Radiol 22:1803-1811, 2012

15. Malagò R, D’Onofrio M, Zamboni GA, Faccioli N, Falconi M, Boninsegna L, et al: Contrast-enhanced sonography of nonfunctioning pancreatic neuroendocrine tumors. AJR Am J Roentgenol 192:424-430, 2009

16. Piscaglia F, Nolsøe C, Dietrich CF, Cosgrove DO, Gilja OH, Bachmann Nielsen M, et al: The EFSUMB guidelines and recommendations on the clinical practice of contrast enhanced ultrasound (CEUS): update 2011 on non-hepatic applications. Ultraschall Med 33:33-59, 2012

17. Rodriguez FJ, Folpe AL, Giannini C, Perry A: Pathology of peripheral nerve sheath tumors: diagnostic overview and update on selected diagnostic problems. Acta Neuropathol 123:295-319, 2012

18. Salameh J, Baran JL, Tick Chong PS: Patient with amyloidoma of the ulnar nerve and salivary glands. Muscle Nerve 40:1054-1058, 2009

19. Saperstein DS, Amato AA, Wolfe GI, Katz JS, Nations SP, Jackson CE, et al: Multifocal acquired demyelinating sensory and motor neuropathy: the Lewis-Sumner syndrome. Muscle Nerve 22:560-566, 1999

20. Scheidl E, Böhm J, Simó M, Rózsa C, Bereznai B, Kovács T, et al: Ultrasonography of MADSAM neuropathy: focal nerve 
enlargements at sites of existing and resolved conduction blocks. Neuromuscul Disord 22:627-631, 2012

21. Van den Bergh PY, Rajabally YA: Chronic inflammatory demyelinating polyradiculoneuropathy. Presse Med 42:e203e215, 2013

22. Yuan MX, Li R, Zhang XH, Tang CL, Guo YL, Guo DY, et al: Factors affecting the enhancement patterns of intrahepatic cholangiocarcinoma (ICC) on contrast-enhanced ultrasound (CEUS) and their pathological correlations in patients with a single lesion. Ultraschall Med [epub ahead of print], 2015

\section{Disclosure}

The authors report no conflict of interest concerning the materials or methods used in this study or the findings specified in this paper.

\section{Author Contributions}

Conception and design: Pedro, Antoniadis, Koenig. Acquisition of data: Pedro, Koenig. Analysis and interpretation of data: Pedro. Critically revising the article: Scheuerle, Pham, Wirtz, Koenig. Neuropathology: Scheuerle.

\section{Correspondence}

Maria Teresa Pedro, Department of Neurosurgery, University of Ulm, Campus Guenzburg, Ludwig-Heilmeyer-Strasse 2, Guenzburg 89312, Germany. email: maria-teresa.pedro@uni-ulm.de. 